INPLASY

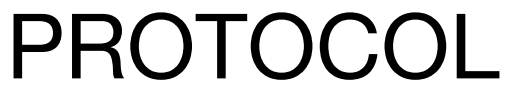

To cite: Liu et al. Efficacy and safety of abdominal acupuncture for knee osteoarthritis: A protocol for systematic review and metaanalysis. Inplasy protocol 2020110020. doi:

10.37766/inplasy2020.11.0020

Received: 05 November 2020

Published: 05 November 2020

Corresponding author:

Liu Min

276816857@qq.com

Author Affiliation:

The First Clinical Medical

School, Guangzhou University

of Chinese Medicine

Support: Hospital

Construction Project.

Review Stage at time of this submission: The review has not yet started.

Conflicts of interest:

The authors have no conflicts

of interest to disclose.

\section{Efficacy and safety of abdominal acupuncture for knee osteoarthritis: A protocol for systematic review and meta-analysis}

Liu, M1; Liu, M²; Zhang, H³; Peng, G4; Sun, X5; Zhu, X6; Zeng, Y7.

Review question / Objective: The purpose of this study is to evaluate the efficacy and safety of abdominal acupuncture for knee osteoarthritis.

Condition being studied: Knee osteoarthritis (KOA) is a disease based on degenerative pathological changes. Most commonly seen in the elderly and is one of the main causes of leg pain, Most patients with knee arthritis have mild symptoms in the beginning, but the condition will get worse if left untreated. The main symptoms include knee pain, swelling and ringing in the knee joint. Stiffness and chills in the knee joint are also symptoms of arthritis, and in severe cases, movement may be limited. If left untreated, it can lead to joint deformity and disability.

INPLASY registration number: This protocol was registered with the International Platform of Registered Systematic Review and Meta-Analysis Protocols (INPLASY) on 05 November 2020 and was last updated on 05 November 2020 (registration number INPLASY2020110020).

\title{
INTRODUCTION
}

Review question / Objective: The purpose of this study is to evaluate the efficacy and safety of abdominal acupuncture for knee osteoarthritis.
Rationale: A large number of clinical studies have reported that abdominal acupuncture has a good effect on the treatment of knee osteoarthritis, but there is no relevant systematic review. 
Condition being studied: Knee osteoarthritis (KOA) is a disease based on degenerative pathological changes. Most commonly seen in the elderly and is one of the main causes of leg pain, Most patients with knee arthritis have mild symptoms in the beginning, but the condition will get worse if left untreated. The main symptoms include knee pain, swelling and ringing in the knee joint. Stiffness and chills in the knee joint are also symptoms of arthritis, and in severe cases, movement may be limited. If left untreated, it can lead to joint deformity and disability.

\section{METHODS}

Search strategy: The following 8 electronic databases will be searched including PubMed, Embase, the Cochrane Library, China National Knowledge Infrastructure (CNKI), Web of Science, Chinese Scientific Journal Database(VIP), Wanfang Database and Chinese Biomedical Literatures Database (CBM) from their inception to 1 November 2020 without any restrictions.

Participant or population: The patients who are diagnosed with Knee osteoarthritis regardless of ethnic group, severity, syndrome type and source of cases clinically will be included. Studies that Knee osteoarthritis combined with other basic diseases will be excluded.

Intervention: The studies that used abdominal acupuncture as the single therapy or as the main part of a combination treatment with other interventions (e.g. western medicine, etc) will be considered. And the studies that abdominal acupuncture was used as an ancillary treatment will be excluded.

Comparator: The control interventions will include: positive interventions (e.g. western medicine), no intervention, placebo or sham moxibustion. The choice of specific forms, as follows:(1).abdominal acupuncture VS positive interventions;(2). abdominal acupuncture + positive interventions VS positive interventions; (3). abdominal acupuncture VS no intervention; (4). abdominal acupuncture VS placebo; (5).abdominal acupuncturevs sham moxibustion.

Study designs to be included: All relevant randomised controlled trials (RCTs) or quasi-RCTs will be included.

Eligibility criteria: All the RCTs or quasiRCTs of abdominal acupuncture for patients with KOA will be included without publication status restriction, review articles, case reports, conference abstracts, cross-sectional studies, and all observational studies will be excluded.

Information sources: We will electronically search the randomized controlled trials in the following databases: including the PubMed, Embase, the Cochrane Library, China National Knowledge Infrastructure (CNKI), Web of Science, Chinese Scientific Journal Database(VIP), Wanfang Database and Chinese Biomedical Literatures Database (CBM) from their inception to 1 November2020 without any restrictions. The search strategy that will be run in the PubMed and adjusted to fit the other database when necessary.

Main outcome(s): 1. The effective rate.2. Visual analogue scale.

Additional outcome(s): 1.The Western Ontario and McMaster Universities Osteoarthritis Index, 2. Incidence of any adverse events.

Quality assessment / Risk of bias analysis: Based on the Cochrane Handbook for Systematic Reviews of Interventions, the risk of bias in all studies will be assessed by 2 authors independently using the Cochrane risk of a bias assessment tool. Six areas of each trial will be evaluated: generation of random sequences, allocation concealment, blinding method, incomplete outcome data, selective reporting and other bias. Each domain will be divided into three levels of bias: unclear risk, high risk and low risk and "Risk of bias" will be filled in. And any differences will be resolved through negotiation or consulting with other reviewers. 
Strategy of data synthesis: We will use RevMan 5.3.0 software to perform the meta-analysis. We will summarize data using risk ratios (RR) with $95 \% \mathrm{Cl}$ for binary outcomes or mean difference (MD) with $95 \% \mathrm{Cl}$ for continuous outcomes. $\mathrm{I}^{2}$ value and $\mathbf{P}$-value will be used to test the degree of heterogeneity. When $P>0.1, I^{2}<50 \%$, no heterogeneity was considered between the studies and the fixed effect model will be used for statistical analysis; otherwise, the random effect model will be used. If there was significant clinical heterogeneity between studies, only descriptive analysis was performed. For a study with incomplete result data, we will try to contact the first author.

Subgroup analysis: In order to explore the possible causes of heterogeneity, we will conduct subgroup analysis if there are a sufficient number of studies (at least 10 trials). In addition, if we do not observe the predicted effect in all the subjects, the subgroup analysis can help us find out whether the treatment is effective in some subgroups.

Sensibility analysis: In order to assure the robustness of our results, We will conduct sensitivity analysis to eliminate the impact of low-quality studies, with the premise of significant heterogeneity still exists right after validation of inputted data and subgroup analysis. We will contrast the results of these two meta-analyses and decide whether to exclude low-quality researches based on impact on the pooled effect size, sample size and strength of evidence. Nevertheless, if all included studies are at high risk of bias, we will not conduct sensitivity analysis.

Language: English.

Country(ies) involved: China.

Keywords: Knee osteoarthritis, abdominal acupuncture, randomized controlled trial, protocol.

Dissemination plans: We have no dissemination plans.
Contributions of each author:

Author 1 - Liu Min - drafted the manuscript. Author 2 - Liu Meinian - provided statistical expertise, write the original draft.

Author 3 - Zhang Haitao - contributed to the development of the selection criteria.

Author 4 - Peng Guanrong - contributed to the risk of the bias assessment strategy.

Author 5 - Sun Xiaobo - provided feedback and approved the final manuscript.

Author 6 - Zhu Xingyang - use the software to analyze the data.

Author 7 - Zeng Yirong - review and edit the manuscript. 